\title{
The Nonlinear Nature of Learning - A Differential Learning Approach
}

\author{
W. I. Schöllhorn*,1, P. Hegen ${ }^{1}$ and K. Davids ${ }^{2}$ \\ ${ }^{1}$ University of Mainz, Institute for Sport Science, Albert Schweitzer Straße 22, 55099 Mainz, Germany \\ ${ }^{2}$ Queensland University of Technology, Australia
}

\begin{abstract}
Traditional learning approaches are typically based on a linear understanding of causality where the same cause leads to the same effect. In recent years there has been increasing interest in the complexity of nature and living phenomena, with significant insights provided by models of change that are based on a nonlinear understanding of causality, where small causes can lead to big effects and vice versa. In this vein, learning processes seem to be more successful for inducing behavioral change when teaching processes deviate from a linear approach. The differential learning approach takes advantage of fluctuations in a complex system by increasing them through 'no repetition' and 'constantly changing movement tasks' which add stochastic perturbations. Previous research has provided much evidence on the superiority of a differential learning approach for learning single movement techniques, in comparison to repetition- and correctionoriented approaches. In this pilot study, the parallel acquisition and learning of two movement techniques in the sport of football are the objective of investigation. One traditionally trained group and two differentially trained groups (blocked and random) trained for 4 weeks, twice a week, on ball control and shooting at goal tasks. Results supported previous work and revealed significant advantages for both differential groups in the acquisition phase as well as in the learning phase, compared to the traditional group. These data suggest that, instead of following a direct linear path towards the target of a 'to-be-learned' movement technique by means of numerous repetitions and corrections, a differential approach is more beneficial because it perturbs learners towards more functional movement patterns during practice.
\end{abstract}

Keywords: Differential Learning, complex systems, fluctuations, football, movement variability.

\section{INTRODUCTION}

Traditional models of learning have recently been questioned because of their principles that all learners typically start with the same exercise followed by other identical teaching exercises in order to build up a methodical sequence of exercises followed by all students in order to achieve stipulated learning goals [1]. A similar logic underpins the interpretation of traditional pedagogical principles that all learners need to progress "from easy to hard" or "from simple to complex" exercises. In principle this logic implies the understanding of linear causality as fundamental basis for a linear pedagogy. In a weak version of this approach to learning, linear causality assumes that same causes will lead to same effects. In the strong version (because much more mathematical conditions have to be fulfilled) similar causes will lead to similar effects. In reality these assumptions are associated with models of linear equations in which the result is just a sum of weighted parameters of influence. In practice this approach is accompanied by the breaking up of a sports movement into certain phases or anatomical focuses that are all trained separately and put together at the end.

From a structural point of view traditional learning approaches in general, and motor learning specifically, can be considered as being similarly based on a few latent

*Address correspondence to this author at the University of Mainz, Institute for Sport Science Albert Schweitzer Straße 22, 55099 Mainz, Germany; Tel: 06131 3923583; Fax: 06131 3920643;

E-mail: schoellw@uni-mainz.de assumptions. Two of these underlying assumptions with the biggest influence on the practical consequences in motor learning are discussed in this paper. These assumptions can be considered as simplified projections of reality on models. One model serves as a 'to-be-achieved target' that is primarily prescribed by an external instructor. The second model is related to the path along which the target movement is to be achieved. In most cases both models imply latent assumptions that restrict possible practical consequences and limit the possible potential of the learning system. Here we discuss these mainly latent assumptions with respect to their plausibility and subsequently we will suggest consequences that can be drawn from slightly revised, but experimentally verified, more plausible assumptions. The consequence of our analysis is to end up with the differential learning approach which considers rather movement variations as the basis of learning than movement repetitions, realizing it by adding stochastic perturbations to a central movement pattern in order to ensure no precise movement repetitions and no corrections during the skill acquisition process [2,3]. Several studies have compared the effectiveness of different interventions on the basis of training single sport techniques $[4,5]$. In summary, the interventions which added stochastic perturbations during learning were at least as successful as traditional training methods. In the majority of cases, the differential learning approach resulted in better skill acquisition and better learning rates in participants $[6,7]$. The practical consequences of the revised assumptions have been tested in a study of skill acquisition in the team sport of football. This football experiment extends previous work since 
two techniques are learned with nonlinear methods in parallel and are compared with a classical linear learning approach.

The first assumption with respect to a to-be-learned target movement can be described by the assumption of a general time independent model that satisfies so called objective criteria for optimum performance. Nominal classic 'textbook' movement, reported in many coaching manuals, can be considered as general because it is provided as a model for all learners to follow without any specifications for differentiation by gender, age or level of performance. Accordingly, textbooks or coaching manuals do not distinguish changes to the to-be-learned target movement on a time scale and, therefore, can be considered as time independent. Independent of the problem of objectivity these performance models are typically derived from research on the world's best athletes and it is unclear whether they can be applied to performance of sub-elite individuals or athletes at the development stage in elite sport.

\section{THE ASSUMPTION OF A GENERAL VALID MODEL}

The first underlying assumption for linear learning is related to the target movement that usually has to be achieved during a learning process. Most often the specific target pattern within a motor learning process is prescribed by the trainer or teacher whose knowledge is typically based on scientific research, which often relies on measures and scores from studies of the world's best athletes. Performance data from groups of world class athletes is typically chosen as the reference system for comparison of athletes.

Previous analyses of the movement patterns of world class male and female javelin throwers have led to an enforced rethink of the traditional way of training fundamentally. The results of these studies revealed the identification of athletes on the basis of a holistic movement description during only a few milliseconds of performance [8]. Beside nationality-specific throwing styles, the recognition procedure led to the identification of highly individual throwing patterns which were stable over several years. Most important, and most critical, for the traditional role of a movement archetype was the additional evidence of no identical movement repetitions and the possibility of throwers performing at world class level with different movement patterns, although they were all within the area of a fictive biomechanical optimum. With no evidence for an identical movement pattern, the repetitive approach in practice is questioned due to the low probability of being confronted with the same performance conditions even after several thousand repetitions. With no evidence for the existence of an optimal movement pattern, the question arises: which top athlete's movement pattern should be copied within a learning process? The individuality of movement patterns has been shown, not only in high performance sports, but also in individuals performing every day movements like walking, where even more repetitions did not lead to the repetition of identical movements [9]. Given these findings, we are confronted with the problem of how to prepare an athlete for the next movement execution which will involve adaptation to unknown environmental conditions. Consequently, it seems that a movement repetition can be divided into a known and an unknown part (Fig. 1). Traditionally, the known part of the movement pattern is considered during the learning progress by ignoring the unknown part or assigning it a perturbing influence due to noise.

Closely related to the difficulty of the characteristics of a to-be-achieved target is the problem of how to bridge the gap between an initial and a target state of a movement outcome. In assumptions of a precise time independent target and a precise initial situation it is plausible to connect the two points linearly and to follow this path in learning. Independent of the uncertainty of the path, an even bigger problem occurs when the target movement is reasonably assumed to be time dependent and different for each individual. Both assumptions increase the uncertainty of the target movement outcome as well. If we divide the trace towards the to-beachieved target into arbitrary sub-units we realize that the problem of uncertainty is accompanied with every sub-goal and leads to an uncertain path overall. Obviously, the practical consequences are highly dependent on the assumptions of the model of the target that are made in advance. Plausible arguments for the assumptions of the uncertainty of a target are given by the identification of individual movement techniques as well as by the low probability of occurrence of two identical movement repetitions. Accordingly, the probability of linear connections between an initial and target situation decreases enormously. Considering not just a single but several subsystems functioning during the same task problem in parallel, and allowing only some interactions between the subsystems, decreases the probability of success in linear learning approaches as well. Despite earlier indications by Bernstein [10] with his statement about "repeating without repetition" and extensive theoretical evidence by Hatze [11], especially within the field of practical applications, variability in movement repetitions has been tended to be considered as destructive. A more constructive interpretation of movement variability emerged with the system dynamic approach followed by comprehensive descriptions and analysis of noise $[12,13]$. Due to methodological reasons the original meaning of noise with its disturbing influence on a signal in general is mostly limited to an equidistant disturbing influence which can be analyzed quantitatively by means of frequency analysis methods. Because learning and training does not usually follow equidistant measurements, the term stochastic perturbation is applied. Meanwhile noise has become an essential component of a few motor control theories [14, $15]$.

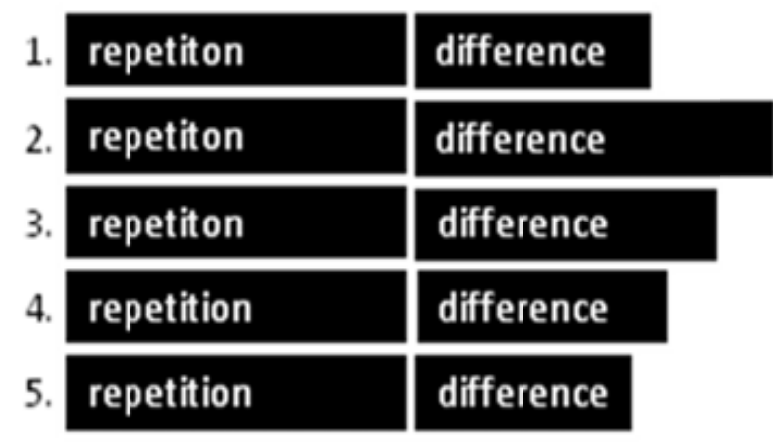

Fig. (1). Differences in the repetition of an assumingly "identical movement". 


\section{DIFFERENTIAL LEARNING}

However, a transfer of the structural idea of noise to practice and therapy programs has been realized in the differential learning approach [16]. The differential learning approach is mainly characterized by taking advantage, for the purpose of learning, of fluctuations that occur, without movement repetitions and without corrections during the skill acquisition process [3]. This approach can be considered as highly nonlinear because of learners constantly performing the whole complex movement with permanently changing stochastic perturbations. In contrast to a nonlinear pedagogical approach, originally suggested by Davids, Shuttleworth and Chow [17] and Chow et al. [18], where key tasks constraints are manipulated in order "to facilitate the emergence of functional movement patterns and decision making behaviors", the differential learning approach does not identify key task constraints. In the differential learning approach the fluctuations in the learner's subsystems itself are exploited during learning, because they have the potential to destabilize the whole system. This destabilization process can lead to an instability that has the advantage of requiring less energy in order to achieve a new stable state of organization for the learner. By amplifying these observed fluctuations the system is additionally confronted with the potential limits of possible performance solutions. Consequently, a self organizing process is initiated and exploited that forces the system to instigate a new coordination strategy which typically results in the emergence of more effective or more stable movement patterns. Although in many sport techniques coarse biomechanical constraints are known and can be assumed to be valid, within a certain level of performance and even at the highest level of performance, infinite solutions are still possible. It, therefore, seems to be hard to identify key constraints that are valid for each individual learner in each performance situation. However, these amplified fluctuations tend to increase fluctuations in other anatomical areas of the body and lead to a highly nonlinear adaptation process. Several experiments have shown higher skill acquisition rates for the differential learning approach in comparison to traditional linear approaches and, most intriguingly, display even better performance improvements in the retention phase of learning $[6,19]$. Recently, a similar influence on physiological parameters in professional cyclists has also been demonstrated by Bauer [4]. Two groups of cyclists aimed to improving the efficiency of cycling movements. One group was supported by specific biomechanical feedback about the deviation of the force that was not perpendicular to the radial movement of the pedals. The second group instead trained with two pedals that could be fixed in an arbitrary relative left to right foot phase. After four weeks of training the differential learning group only improved their maximum heart rate level to the highest level of a fourlevel stress test significantly from $178 \mathrm{bpm}$ to $164 \mathrm{bpm}$ and lowered the maximum lactate production in the same test from $7.6 \mathrm{mmol} / 1$ down to $4,8 \mathrm{mmol} / 1$.

However, in all experiments the objective was the improvement of a single technique. Because in different sports, especially team sports, multiple techniques are necessary for success we decided to compare the influence of two techniques trained in parallel: linearly and nonlinearly. The objective of this pilot-study is to compare a classical (linear) training approach with the differential (nonlinear) learning approach during the training of two techniques in football. The single classical approach followed the repetitive and corrective philosophy during learning, while the differential approach was distinguished by different levels of variation. In accordance with the contextual interference debate [20], one approach was differentially training the two techniques in separate blocks of trials, whereas in the second differential approach, the two techniques followed a random order of trial practice. Therefore, the null hypothesis was that all three approaches would not differ with respect to the outcomes in post and retention tests.

\section{METHODS}

Following research that has demonstrated the positive effects of the differential learning approach when observing a single technique in isolation, this study combined two techniques that are essential in football within one training session: movements for shooting a ball and for controlling it. For this purpose, a pre-post test-design with a subsequent retention test was applied.

\section{Participants}

The participants played in the $8^{\text {th }}$ division of the German football league and typically trained twice a week for $2 \mathrm{hrs}$. 24 participants were randomly assigned to three groups. Because only 12 participants were able to participate in every test and training session, the learning processes of 4 participants per group could be observed. More details, including the average age and average football experience as well as the playing position of participants in the three groups, are displayed in Table 1. All participants agreed to take part in the experiment through informed consent.

\section{Protocol}

The pre-, post-, and retention-tests examined the ability of the sample to control a ball within a minimum amount of space [21] and the ability to shoot a ball with precision at goal [21].

Table 1. Average age, Average Football Experience, and Players Field Specialty of the Three Experimental Groups

\begin{tabular}{|c|c|c|c|}
\hline & Average Age [Years] & Football Experience [Years] & $\begin{array}{c}\text { Players Field Specialty of the Four Partici- } \\
\text { pants }\end{array}$ \\
\hline \hline Control group (CG) & $23,8 \pm 3,9$ & $18,5 \pm 4,7$ & 1 defender and 3 midfielders \\
\hline Differential blocked group (DBG) & $24,5 \pm 2,1$ & $20,8 \pm 3,4$ & 2 defenders, 1 midfielder and 1 striker \\
\hline Differential random group (DRG) & $24,5 \pm 2,1$ & $20,5 \pm 1$ & 2 defenders and 2 midfielders \\
\hline
\end{tabular}


In the ball-control test a ball was thrown towards the participants in a parabolic flight path from an assistant who was trained and tested to throw with a certain constancy towards a given target. The thrower was not informed about the objective of the experiment. The ball was thrown: a) in a direct parabolic flight at a height that forced the participant to receive the ball first with the chest; and b), with a shorter parabolic flight that led to the reception of a bounced ball (Fig. 2).

In the goal shooting test, participants had to shoot the ball at goal without a goal keeper from the $16 \mathrm{~m}$ line in 7 different situations, with each situation repeated 5 times. Each participant performed overall 35 shooting movements in a blocked order ( 7 situations $\times 5$ times $=35$ trials $)$. The seven different goal shooting situations were [22](Fig. 3):

1. Five immobile balls were shot towards the goal after a short run-up from position 1.

2. Five balls were shot towards the goal after a $10 \mathrm{~m}$ dribbling from position 1 .

3. Five balls were shot towards the goal after a $5 \mathrm{~m}$ dribbling from position 2 .

4. Five balls were shot towards goal from position 1 after a pass from the right.

5. Five balls were shot towards the goal after a $5 \mathrm{~m}$ dribbling from position 3 .
6. Five balls were shot towards the goal from position 1 after a pass from the left.

7. Five balls were shot towards the goal from position 1 after crossing an obstacle of $40 \mathrm{~cm}$ height with a vertical jump.

The whole time schedule for the three tests and the intervention period is depicted in Table 2 .

\section{Data Acquisition}

The quality of ball control was recorded by measuring the distance of the initial contact with the foot and the resting position of the ball after control. Each reception technique was repeated five times in a blocked sequence.

The precision of the shots was measured by dividing the goal into scoring zones. The zones were determined according to plausible probability of scoring a goal. Areas that were hard to be reached by the goalkeeper were scored higher and vice versa. Shots that closely missed the goal scored with 1 point still (Fig. 4).

\section{Training Intervention}

A pre-test (Figs. 2 and 3) was followed by a four-week training intervention. For four weeks, within the normal club training program, the training intervention consisted of eight sessions (two per week). In all eight sessions 20 exercises for

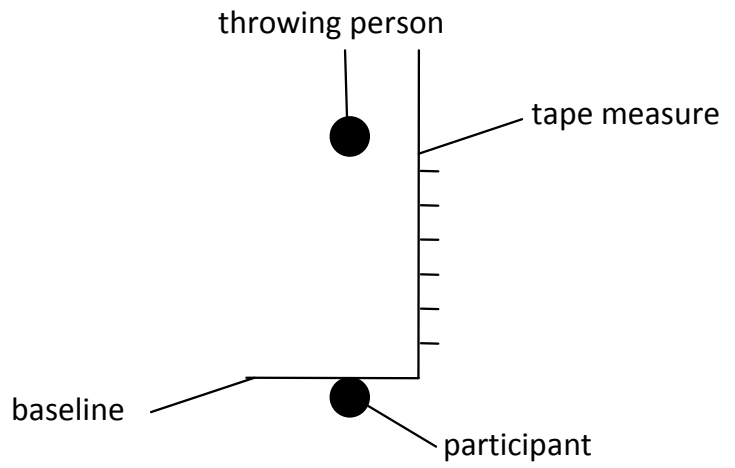

a.)

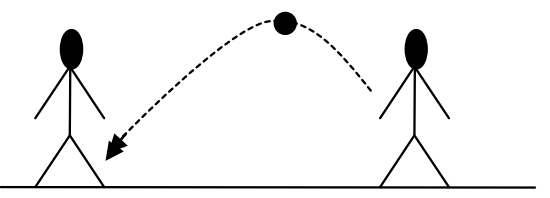

b.)

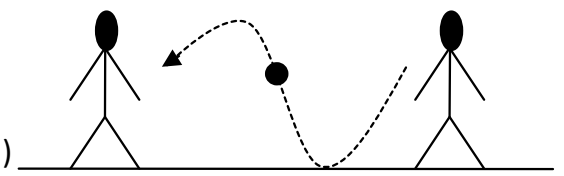

Fig. (2). Experimental set-up from the bird's eye perspective to determine the quality of the ball's reception (left). Direct throw of the ball (a.) ball's reception with the foot and indirect throw via bottom contact (b.) ball's reception with the chest.

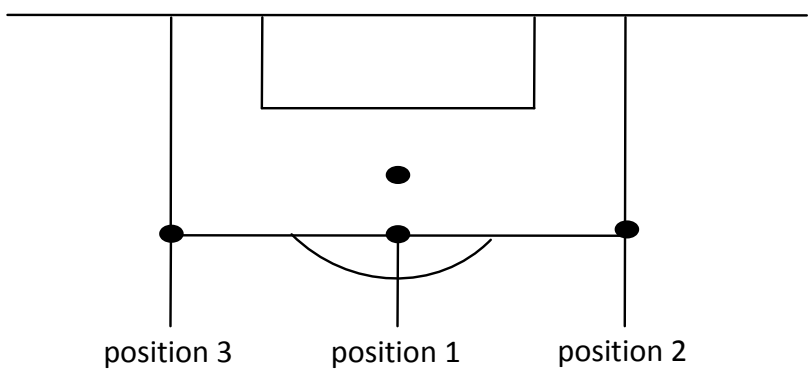

Fig. (3). Initial goal shooting positions with different tests.

Table 2. The Intervention Schedule

\begin{tabular}{|c|c|c|c|c|}
\hline Pre-Tests & Training Interventions & Post-Test & Break - no Training & Retention Test \\
\hline \hline $1^{\text {st }} w e e k$ & $2^{\text {nd }}-5^{\text {th }}$ week & $6^{\text {th }}$ week & $7^{\text {th }}-8^{\text {th }}$ week & $9^{\text {th }} w e e k$ \\
\hline
\end{tabular}




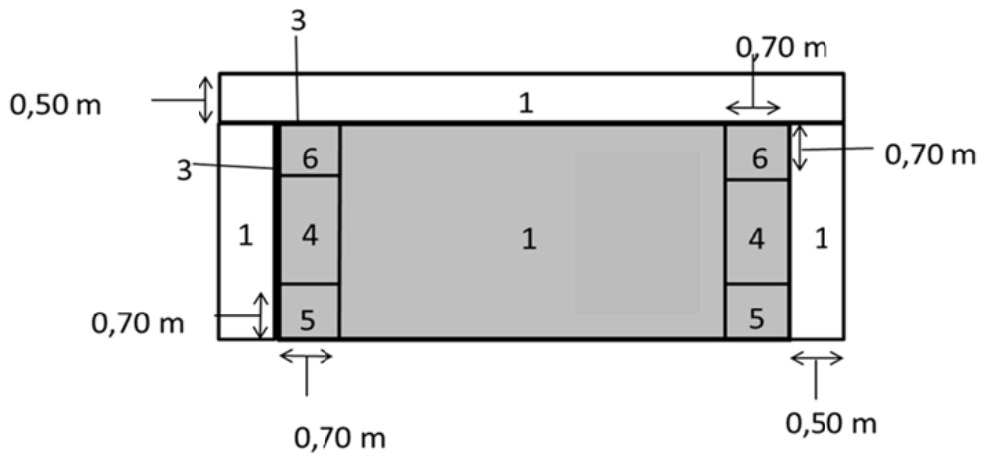

Fig. (4). Division of the goal in different areas with the scores 1, 3, 4, 5, 6 and the corresponding measures for each zone.

Table 3. Exemplary Trainings Plan for the CG with Orientation on the Ideal Movement Archetypes

\begin{tabular}{|l|l|l|l|}
\hline No. & \multicolumn{1}{|c|}{ Exercises } & \multicolumn{1}{c|}{ Repetition } & \multicolumn{1}{c|}{ Explanation } \\
\hline \hline 1 & Goal shot with fixed ball & 10 & $\begin{array}{l}\text { 1. The supporting leg one foot beside the ball. } \\
\text { 2. The shot leg swings linear to the ball. } \\
3 . \text { The ankle is craned and fixed } \\
\text { 4. The upper body is over the ball. } \\
\text { 5. The ball should be crossed in center. }\end{array}$ \\
\hline 2 & Ball reception with the chest & 10 & $\begin{array}{l}\text { 1. The upper body draw back when the ball is at the chest. } \\
\text { 2. Control the ball with the foot on the ground. }\end{array}$ \\
\hline 4 & Goal shot in motion & 10 & $\begin{array}{l}\text { 1. The supporting leg one foot beside the ball. } \\
\text { 2. The shot leg swings linear to the ball. } \\
\text { 3. The ankle is craned and fixed } \\
\text { 4. The upper body is over the ball. } \\
\text { 5. The ball should be crossed in center. }\end{array}$ \\
\hline
\end{tabular}

Order of the exercises

Explanation of the technique goal shot

10 goal shots

Explanation of the ball reception with the chest

10 ball receptions

Explanation of the technique goal shot

10 goal shots

Explanation of the ball reception with the foot

10 ball receptions

Important: Corrections 5 seconds after completing the task and only every third trial!

the goal shooting technique and 20 exercises for the ball control technique were performed. Each intervention lasted about 25 minutes. In summary, each participant performed a total of 160 exercises for both techniques. The CG trained according to the classical training approach oriented on ideal movement archetypes for goal shooting and ball control movements (Table 3). Both techniques were trained in a blocked order: the goal shooting technique in the first half of the training session followed by the ball control task in the second half. Methodological sequences of exercises on goal shots and ball's control with numerous repetitions and error corrections were conducted. Criteria for the optimum performance of the goal shooting movement included the position of the standing leg, orientation of the head, amplitude of the kicking leg, sequence of the maximum velocity in the limbs of the kicking leg, stiffness of the kicking leg at ball contact and arm movements during the approach and during the kicking movement. Main criteria for functional technique in ball reception were fixation of the approaching ball, soft first ball contact.

The two other groups trained (DBG and DRG) according to the differential learning approach, one group with both techniques in blocked order and the other group with random order in one training session (see Tables 4 and 5). The core idea of the two differential training groups was to increase the fluctuations of both techniques in order to make the athletes more stable against disturbances and in order to provide the athletes the possibility to seek and explore functional movement patterns. The fluctuations were increased by infi- 
nite variations in each technique as well as by avoiding movement repetitions and by providing no corrective feedback. Movement variations were characterized by variations in the standing leg, in the kicking leg, in the arms, in the trunk, in the head and the ball, referring to the angles, the angular velocity and the rhythm of each joint movement. To exemplify: the standing leg had to be placed well before the ball, well behind it, or well to the side of the ball; the knee joint in one shot had to be stiff, in the other it was kept very flexible or alternating between stiffness and flexibility after each shot.

\section{Table 4. Exemplarily Trainings Plan for the DBG}

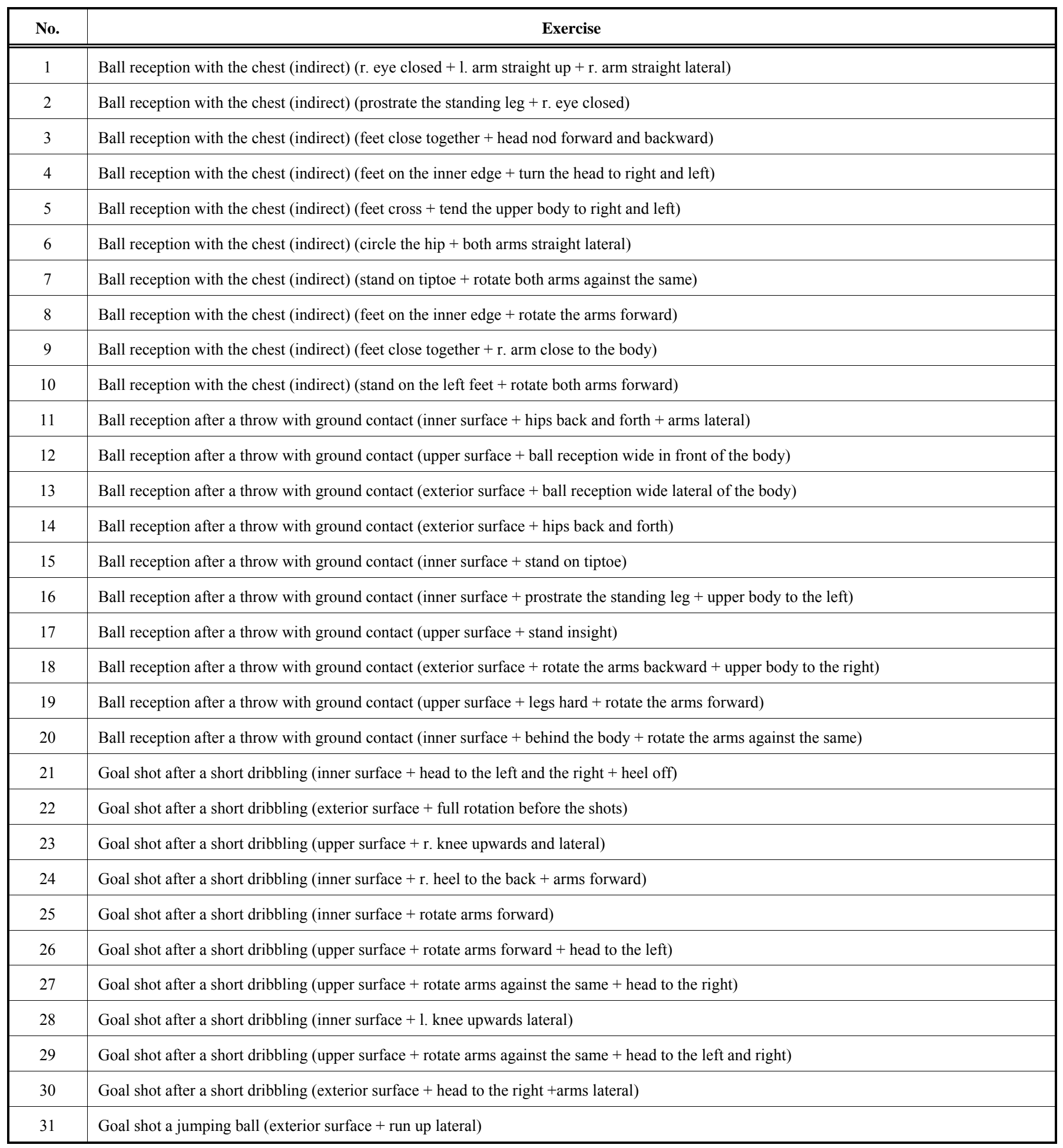




\begin{tabular}{|c|l|}
\hline No. & \multicolumn{1}{|c|}{ Exercise } \\
\hline \hline 32 & Goal shot a jumping ball (upper surface + upper body to the right + run up with little jumps) \\
\hline 33 & Goal shot a jumping ball (inner surface + run up cross + both arms up) \\
\hline 34 & Goal shot a jumping ball (exterior surface + circle the hip + bot arms up) \\
\hline 35 & Goal shot a jumping ball (upper surface + both arms forward + hip to the left and right) \\
\hline 36 & Goal shot a jumping ball (inner surface + sidesteps; + head nod forward and backward) \\
\hline 37 & Goal shot a jumping ball (exterior surface + standing leg wide beside the ball + eyes blinking) \\
\hline 38 & Goal shot a jumping ball (inner surface + standing leg before the ball + eyes blinking) \\
\hline 39 & Goal shot a jumping ball (inner surface + run up with jumps + arms lateral) \\
\hline 40 & Goal shot a jumping ball (exterior surface + upper body forward and backward) \\
\hline
\end{tabular}

\section{Table 5. Exemplarily Trainings Plan for the DRG. The Different to the DGB is the Order of the Exercises}

\begin{tabular}{|c|c|}
\hline No. & exercise \\
\hline 1 & Ball reception with the chest (indirect) $(r$. eye closed +1. arm straight $u p+r$. arm straight lateral) \\
\hline 2 & Ball reception with the chest (indirect) (prostrate the standing leg $+r$. eye closed) \\
\hline 3 & Ball reception with the chest (indirect) (feet close together + head nod forward and backward) \\
\hline 4 & Ball reception with the chest (indirect) (feet on the inner edge + turn the head to right and left) \\
\hline 5 & Ball reception with the chest (indirect) (feet cross + tend the upper body to right and left) \\
\hline 6 & Ball reception with the chest (indirect) (circle the hip + both arms straight lateral) \\
\hline 7 & Ball reception with the chest (indirect) (Stand on tiptoe + rotate both arms against the same) \\
\hline 8 & Ball reception with the chest (indirect) (feet on the inner edge + rotate the arms forward) \\
\hline 9 & Ball reception with the chest (indirect) (feet close together $+r$. arm close to the body) \\
\hline 10 & Ball reception with the chest (indirect) (stand on the left feet + rotate both arms forward) \\
\hline 11 & Ball reception after a throw with ground contact (inner surface + hips back and forth + arms lateral) \\
\hline 12 & Ball reception after a throw with ground contact (upper surface + ball reception wide in front of the body) \\
\hline 13 & Ball reception after a throw with ground contact (exterior surface + ball reception wide lateral of the body) \\
\hline 14 & Ball reception after a throw with ground contact (exterior surface + hips back and forth) \\
\hline 15 & Ball reception after a throw with ground contact (inner surface + stand on tiptoe) \\
\hline 16 & Ball reception after a throw with ground contact (inner surface + prostrate the standing leg + upper body to the left) \\
\hline 17 & Ball reception after a throw with ground contact (upper surface + stand insight) \\
\hline 18 & Ball reception after a throw with ground contact (exterior surface + rotate the arms backward + upperbody to the right) \\
\hline 19 & Ball reception after a throw with ground contact (upper surface + legs hard + rotate the arms forward) \\
\hline 20 & Ball reception after a throw with ground contact (inner surface + behind the body + rotate the arms against the same) \\
\hline 21 & Goal shot after a short dribbling (inner surface + head to the left and the right + heel off) \\
\hline 22 & Goal shot after a short dribbling (exterior surface + full rotation bevor the shots) \\
\hline 23 & Goal shot after a short dribbling (upper surface + r. knee upwards and lateral) \\
\hline 24 & Goal shot after a short dribbling (inner surface $+r$. heel to the back + arms forward) \\
\hline 25 & Goal shot after a short dribbling (inner surface + rotate arms forward) \\
\hline
\end{tabular}


Table 5. Contd......

\begin{tabular}{|c|c|}
\hline No. & Exercise \\
\hline 26 & Goal shot after a short dribbling (upper surface + rotate arms forward + head to the left) \\
\hline 28 & Goal shot after a short dribbling (inner surface +1 . knee upwards lateral) \\
\hline 29 & Goal shot after a short dribbling (upper surface + rotate arms against the same + head to the left and right) \\
\hline 31 & Goal shot a jumping ball (exterior surface + run up lateral) \\
\hline 32 & Goal shot a jumping ball (upper surface + upper body to the right + run up with little jumps) \\
\hline 33 & Goal shot a jumping ball (inner surface + run up cross + both arms up) \\
\hline 37 & Goal shot a jumping ball (exterior surface + standing leg wide beside the ball + eyes blinking) \\
\hline 38 & Goal shot a jumping ball (inner surface + standing leg before the ball + eyes blinking) \\
\hline 39 & Goal shot a jumping ball (inner surface + run up with jumps + arms lateral) \\
\hline 40 & Goal shot a jumping ball (exterior surface + upper body forward and backward) \\
\hline
\end{tabular}

Order of the excercises for DRG:

$33,17,6,15,2,11,16,3,1,19,9,12,26,23,35,5,37,32,27,31,10,8,14,40,28,13,24,30,4,38,39,21,34,20,18,29,25,22,36,7$

\section{Statistical Analysis}

Nonparametric tests were used to analyze the data. The statistical tests selected for use were especially developed for clinical experiments with low case numbers involving rare diseases. They included the H-Test from Kruskal and Wallis, followed by a single comparison test [23]. The H-Test results in $\mathrm{H}$-values $(\mathrm{H})$ comparable to t-values in the t-test. Once the $\mathrm{H}$-values are below a critical value $\left(\chi^{2}\right)$ the results are considered to be significant. The first test provides information about the global trend and the second test compares the individual group results. At the comparison test the calculated values are $D_{\text {emp }}$ and the critical values for the decision of significance are $\mathrm{D}_{\text {crit }}$. The significance level for both tests was set to 0.05 .

\section{RESULTS}

The results of the pre-, post- and retention-test of the three training groups are displayed in Fig. (5) for the shooting movement and in Fig. (6) for the reception movement. The results of the ball's reception test within the post-test did not reveal any significant difference in the global trend between the three groups $\left(\mathrm{H}=0.76<\chi_{\mathrm{t}}^{2}=5.69\right)$. A significant difference $(\alpha=0.05)$ can be discerned when comparing the classical group and both differential groups separately $\left(D_{\text {emp }}\right.$ $=7.5>\mathrm{D}_{\text {crit }}=5.27$ ). No significant difference could be identified between both differential trained groups $\left(D_{\text {emp }}=2<\right.$ $\mathrm{D}_{\text {crit }}=4.13$ ). The retention-test data analysis of the ball's reception test did not indicate statistically significant differences in the global trend between the three groups $(\mathrm{H}=1.86$ $\left.<\chi^{2}=5.62\right)$. When comparing the three groups separately, a significant difference between the classical group and the two differential groups can be identified $\left(D_{\text {emp }}=12>D_{\text {crit }}=\right.$ 5.27). The comparison between the differential trained groups 1 and 2 does not reveal a significant difference $\left(D_{\text {emp }}\right.$ $=0<D_{\text {crit }}=5.27$ ). Table 6 and Fig. (5) illustrate the data and their time course.

The test results of the goal shooting test reveal a significant global trend $\left(H=7.38>\chi^{2}=5.69\right)$. When comparing the results of the classic trained group and both differential trained groups a statistical difference can be stated again $\left(D_{\text {emp }}=24>D_{\text {crit }}=5.27\right)$. The test results of both differential trained groups were clearly different to the results of the classic trained group. A relevant difference between the differential trained groups cannot be detected $\left(D_{\text {emp }}=0<D_{\text {crit }}=\right.$ 4.13). The statistical analysis of the goal shot test points out a significant difference between the three groups in the global trend $\left(H=7.87>\chi^{2}=5.62\right)$. Again the individual comparison shows a significant difference between the classic trained group and both differential trained groups $\left(D_{\text {emp }}=24\right.$ $>\mathrm{D}_{\text {crit }}=5.27$. . A significant difference can also be determined when comparing the two differential trained groups. Thus the performance of the differential trained group DRG is significantly better than the performance of the differential trained group DBG $\left(D_{\text {emp }}=7>D_{\text {crit }}=4.13\right)$. Table 7 and Fig. (6) show the data and the course. Table 8 gives an overview on all results. Hereby the standard deviations of the goal shooting results have to be interpreted carefully because of the nonlinearity of the chosen scoring.

In summary, the results of the study revealed a significant difference between the $\mathrm{CG}$ and both the differential trained groups (DBG and DRG), with respect to the tasks of ball control and ball shooting (Table 8). Only in the reten- 
Table 6. Mean and Standard Deviation of the Pre-, Post-, and Retentiontest Results of the Reception Task

\begin{tabular}{|c|c|c|c|}
\hline & pre-test (Mean \pm STD) [cm] & post-test (Mean \pm STD) [cm] & retention-test (Mean \pm STD) [cm] \\
\hline \hline Control group (CG) & $654 \pm 308$ & $465 \pm 142$ & $506 \pm 153$ \\
\hline Differential blocked group (DBG) & $568 \pm 192$ & $424 \pm 152$ & $418 \pm 144$ \\
\hline Differential random group (DRG) & $660 \pm 222$ & $416 \pm 143$ & $393 \pm 115$ \\
\hline
\end{tabular}

Table 7. Mean and Standard Deviation of the Pre-, Post-, and Retentiontestresults of the Goal Shooting Task

\begin{tabular}{|c|c|c|c|}
\hline & pre-test (Mean \pm STD) [score] & post-test (Mean \pm STD) [score] & retention-test (Mean \pm STD) [score] \\
\hline \hline Control group (CG) & $32 \pm 9,3$ & $29,5 \pm 1,3$ & $30,8 \pm 4,6$ \\
\hline Differential blocked group (DBG) & $32,5 \pm 4,8$ & $39,8 \pm 1$ & $41 \pm 3,4$ \\
\hline Differential random group (DRG) & $41 \pm 3,6$ & $42,8 \pm 14$ & $45 \pm 6,1$ \\
\hline
\end{tabular}

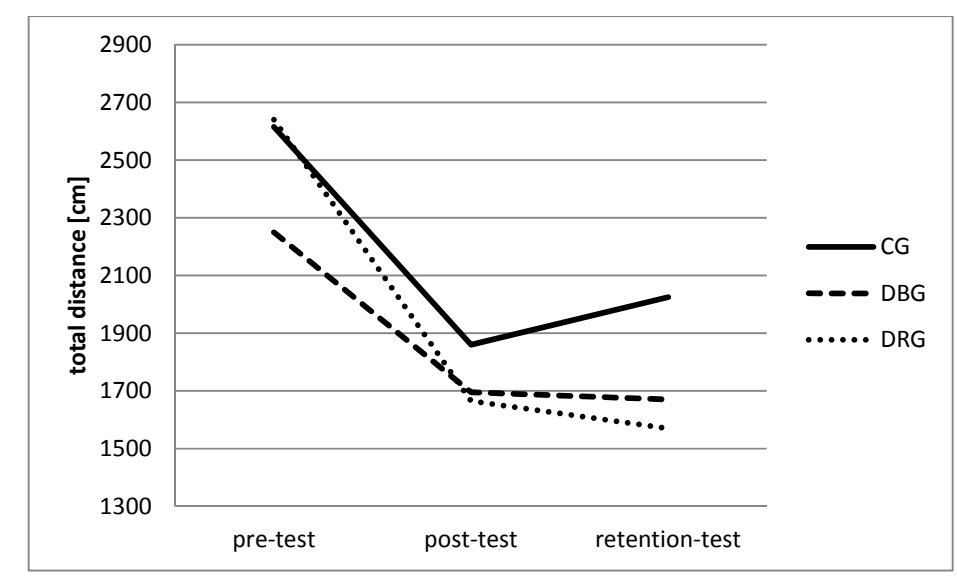

Fig. (5). Pre-, Post-, and Retentiontest of the reception performance.

Note: The total distance is fomed by the sum of the individual participants at five receptions with the foot and five receptions with the chest and subsequentliy with the foot.

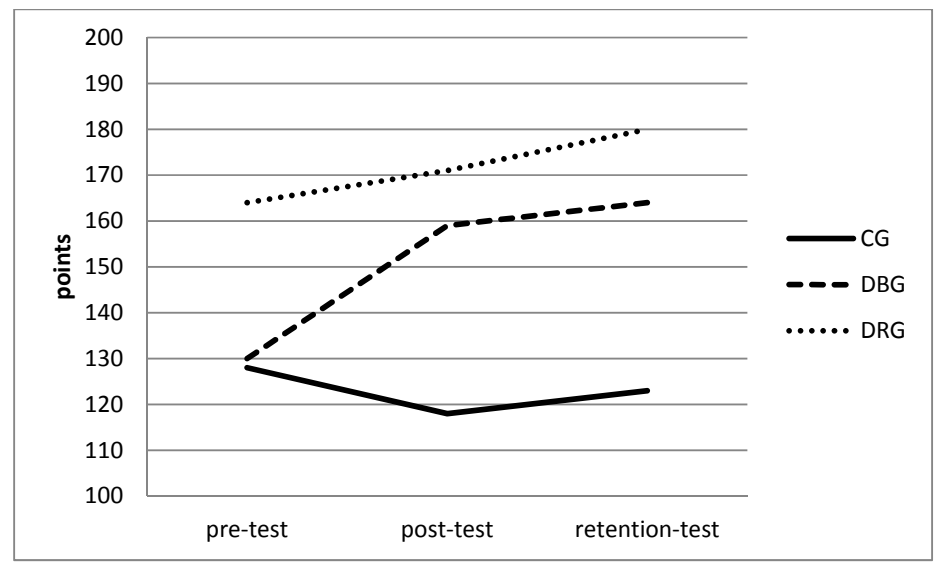

Fig. (6). Pre-, Post-, and Retentiontest scores of the goal shooting performance.

tion-test of the goal shooting technique was a significant difference between the DBG and the DRG groups also observed. An interesting observation is that the DRG was exposed to more fluctuations than the DBG.

Because the differential learning approach supports performance individuality the results of the individual partici- pants were analyzed visually as well [16]. Figs. (7 and 8) display the individual results in all tests. Three out of four participants in the classical group improved their performance within the ball's control test from pre- to post-test (Fig. 7a). Only one participant was not able to keep the starting level. Because this participant had already started at the 
Table 8. Summary of the Significant Test Results between the Groups

\begin{tabular}{|c|c|c|c|c|}
\hline \multirow{2}{*}{} & \multicolumn{2}{|c|}{ post-test } & \multicolumn{2}{c|}{ retention-test } \\
\cline { 2 - 5 } & ball's reception & goal shot & Sall's reception & goal shot \\
\hline \hline \multirow{2}{*}{ CG } & Sig. to DBG & Sig. to DBG & Sig. to DRG \\
& Sig. to DRG & Sig. to DRG & Sig. to CG & Sig. to DBG \\
\hline \multirow{2}{*}{ DBG } & Sig. to CG & Sig. to CG & Sig. to CG \\
& & & Sig. to DRG \\
\hline DRG & Sig. to CG & Sig. to CG & Sig. to CG \\
& & & Sig. to DBG \\
\hline
\end{tabular}

highest performance level, he may have been demonstrating decreasing progress with increasing levels of performance. From the differential learning point of view in this case the fluctuations were decreasing with the number of trial repetitions as performance was increasing and it becomes increasingly harder to enlarge the area of possible solutions that are more successful. The largest performance increase was $45 \%$ achieved by the participant with the lowest starting level. After the two-weeks of break in the retention phase the results of all participants from the classic trained group decreased in the retention-test. This observation corresponds to classical memory effects that follow learning procedures that are mainly characterized by performance repetitions. In comparison all participants of the differential blocked and differential random group improved their performance or kept the level of performance independent of the starting level from the pre- to the post-test. In contrast to the classical group, three out of four participants were able to increase or equalize their posttest level in the retention test. Finally, all participants ended up with increased performance levels in comparison to the pretest. Most intriguingly, in the differential random group, all participants improved their performance in all tests in comparison to the pretest independent of their starting level. There were similar reactions during the retention phase, three participants even improved their performance after the break, and one maintained it. The results indicate that in both differentially trained groups the majority of the participants were able to improve their performance in all tests following the pretest, independent of the initial level, indicating additional evidence for supporting the effect of individuality by the differential learning approach.

Within the goal shooting test only two out of four in the classical group were able to improve performance from preto post-test, the other two participants even decreased their pretest result. Participant 4 had the largest performance increase of $36 \%$. When comparing the results of the post- and the retention-test it can be noticed that three participants improved and one worsened.

The test results of the differential blocked (Fig. 8b) and random (Fig. 8c) group were more homogeneous than the results of the classic trained group. All participants within the DBG improved their goal shooting performance from pre- to posttest. Therefore, three out of four participants were even able to increase the ability for precise kicks in the retention test. In the DRG the most heterogeneous results can be

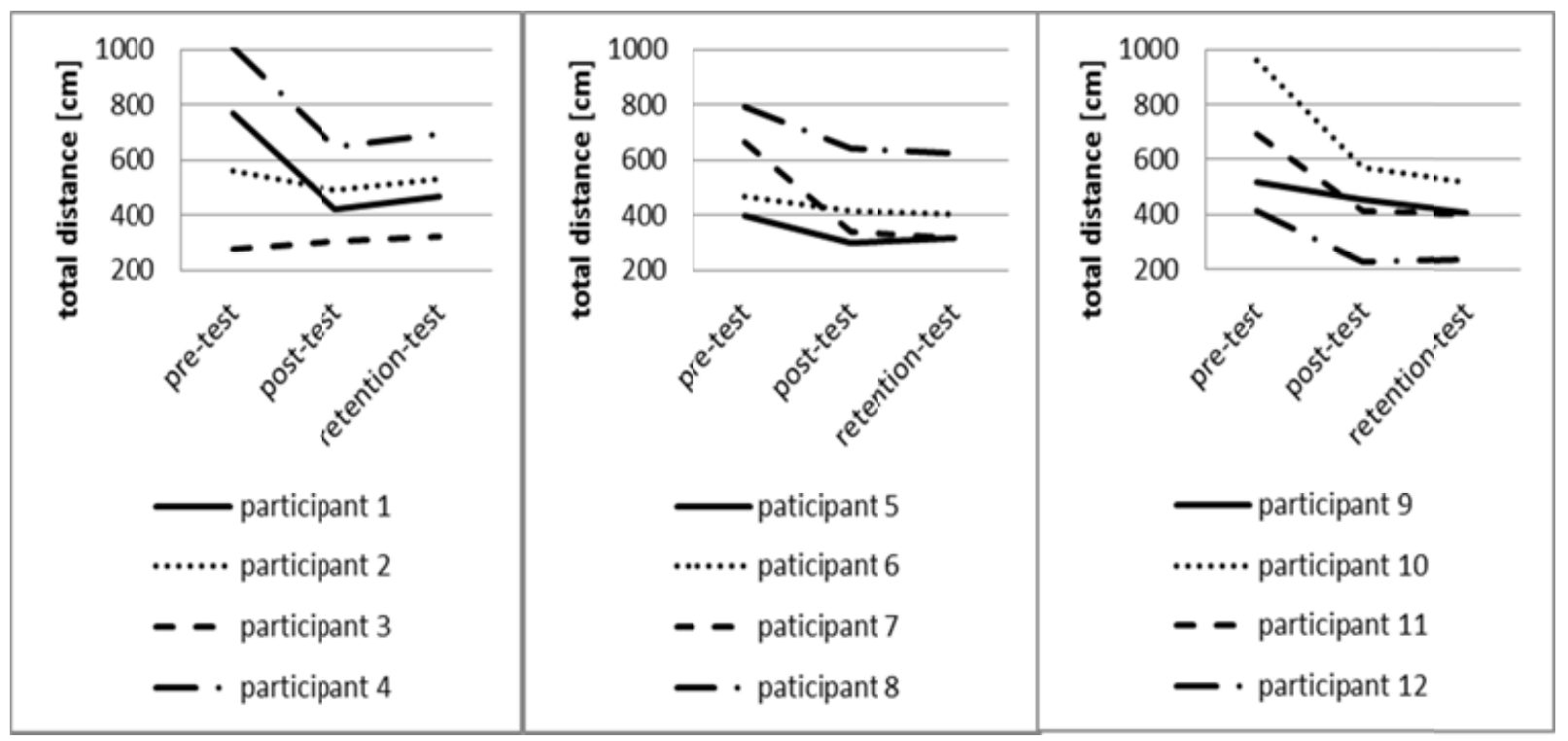

Fig. (7). Performance of test persons regarding the development within the ball's reception test: CG (left), DBG (middle) and DRG (right). 


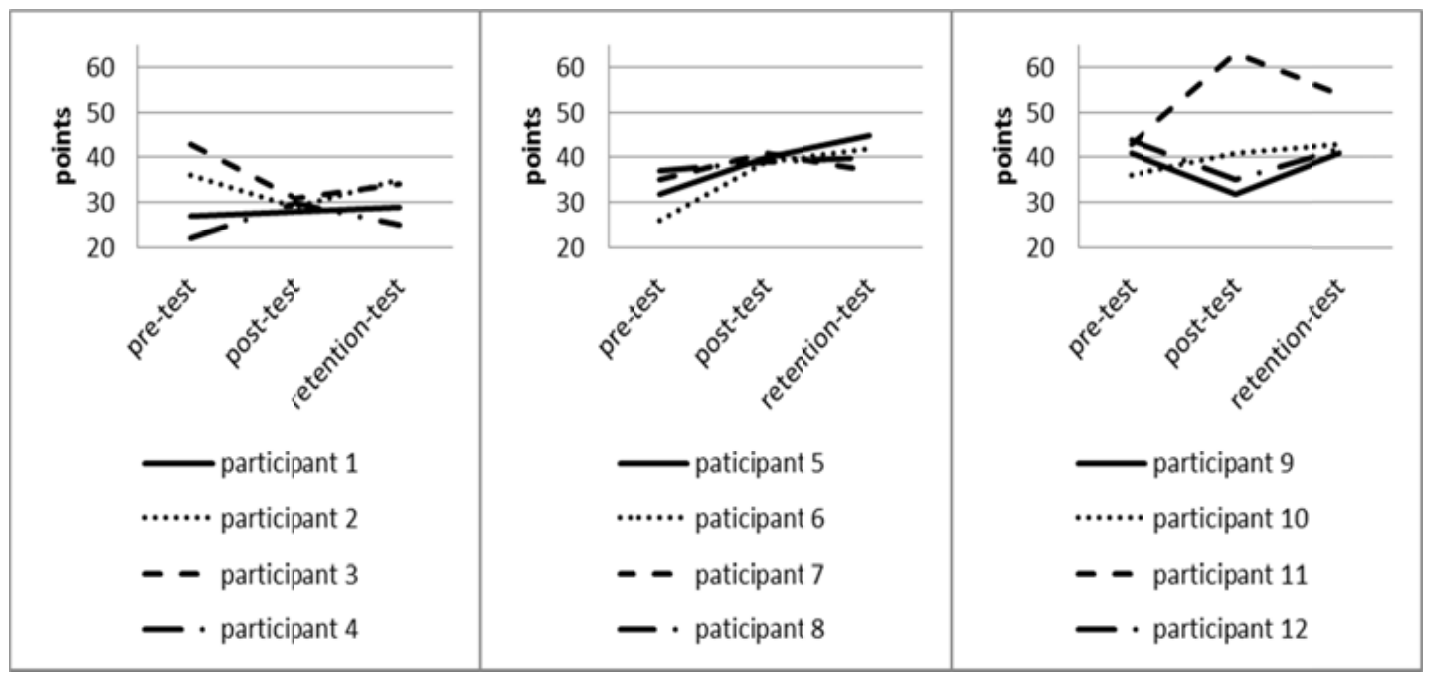

Fig. (8). Performance of test persons regarding the development within the goal shot test: CG (left), DBG (middle) and DRG (right).

observed. Two participants improved their performance with in the first interval whereas the other two even decreased their level. In the subsequent retention phase the two participants who decreased the test score in the first interval increased the test score during the second. The participant who had the largest increase during the first interval decreased his performance during the second interval. Only one athlete could show an increase in performance in both intervals.

\section{DISCUSSION}

In contrast to a classical repetition and correctionoriented teaching methodology, a nonlinear pedagogical approach to teaching two football techniques was investigated in this study. The training intervention had to be included into the normal training program and life schedule of the members of the football club and, therefore, provided a high external validity. Unfortunately, only $50 \%$ of the participants were able to complete their participation in all test and intervention events. The small number of participants gave the study the character of a pilot study. Because of the similar ages and football experience levels of the participants, the groups can be considered as homogenous. Due to the number of participants the individual results in all tests were especially important in this study. Considering the results of both technique tests together, for groups as well as for individuals, offers clear evidence for the superiority of the differential learning approach in comparison to the classical training approach. The results of the classical group and its individuals can be interpreted traditionally [24] as follows. In most cases an increase during the acquisition phase is followed by a decrease in the learning phase during the retention test. In contrast to the classical group, both differential training groups in general showed a clear advantage in learning two techniques in parallel. Both differential groups showed, on average, an increase in performance during the acquisition period, and at least maintenance of performance at the posttest level. In the majority of participants, an increase in performance during the retention phase can be observed as well.
Taking the individual results into account as well, only one participant in the DRG group during the ball reception tests showed extraordinary differences in behavior, compared to the rest of the group. In this case the group results have to be interpreted with care. In comparison to data from earlier studies on differential learning of singular sport techniques, the present results verify the observed tendencies. The tendency shows an increase in learning and skill acquisition rate when differences between two subsequent movements are exploited during training. Because both differentially trained groups ended up with comparable improvements, further research is demanded for the optimum amount of differences that would be functional during training. In addition, these results need to be verified with larger and other samples because in the present study design only the probability of data with the assumption of a true hypothesis was tested but not the hypothesis it self. In this context it has to be mentioned from a stochastic and epistemological point of view that not only the size of sample is of interest in future but rather the number of investigations related to the research topic, and therefore the number of hypothesis in order to pursue the question for the probability of a hypothesis $(=\mathrm{p}(\mathrm{H} 0))[25$, 26].

Somewhat surprising is the higher learning rate of the differential random group, despite their higher initial performance level. But this observation was in accordance with earlier findings in which the more advanced participants responded even acutely to the differential learning approach. In supplementing earlier research, results of the present pilot study of differential learning led to improved skill acquisition and learning rates when participants were confronted with two techniques to learn. How sensitive these results are with respect to the relative similarity of the two to-belearned techniques is a question that needs further research. With respect to the individual results, the differential blocked group showed the most consistent performance. All participants were able to improve their performance in both tests and in both techniques. In comparison the differential 
blocked group only showed homogenous improvements over all three tests in the ball control task, whereas in the goal shooting task, two participants were not able to increase their overall score in comparison to the initial state. Obviously, only some participants could take advantage of the increased system variability to enhance performance and learning. Perhaps for these participants the optimum amount of system variation needed for learning was exceeded.

\section{CONCLUSION}

Based on the theoretical considerations of the differential learning approach and the empirical results observed, a nonlinear learning process can be assumed, with important implications for practice. The differential learning approach exploits these ideas and puts them into practice. After having proven the effectiveness and efficiency of an isolated technique in various sports, this pilot study extends the differential learning approach to two techniques within one training session. The practical consequence of the study presented is that two techniques can easily be trained within one training session by applying the differential learning approach. Negative influences regarding both techniques, like decrease in performance or boredom were not observed, implying that skill and performance in both techniques was developed in a positive way.

Regarding the sequence of exercises it can be stated that there is merely a difference between both differential trained groups. The results showed that it makes no difference whether the exercises are trained en bloc or random. Within one training session two differential training blocks for two separate techniques may well be applied. A switch between the two techniques at random has only caused a statistically significant positive effect within the goal shooting test. Consequently, it can be assumed that this procedure has only a minimal positive effect on memory performance respectively learning capacity of the participants. The participants who constantly switched between both techniques could achieve better goal shooting test results at the retention-test. The results at the ball's reception test do not differ between the two differential trained groups. Due to these findings a distinct statement regarding a recommendation for an exercise sequence cannot be made.

Either way, both differential trained groups performed significantly better than the classic trained group. Due to the nonlinearity of motor learning it seems reasonable to expedite training with stochastic perturbations. Monotonous repetitions of movements should be abandoned whereas large variations should be produced in order to initiate selforganization so that a more effective and more efficient learning process can be designed.

\section{CONFLICT OF INTEREST}

The authors confirm that this article content has no conflicts of interest.

\section{ACKNOWLEDGEMENT}

Declared none.

\section{REFERENCES}

[1] Davids K, Chow JY, Shuttleworth R. A constraints-based framework for nonlinear pedagogy in physical education. J Phys Educ NZ 2005; 38: 17-29.

[2] Schöllhorn WI. Applications of systems dynamic principles to technique and strength training. Acta Acad Olympiquae Est 2000; 8: 67-85.

[3] Schöllhorn WI, Mayer-Kress G, Newell KM, Michelbrink M. Time scales of adaptive behavior and motor learning in the presence of stochastic perturbations. Hum Mov Sci 2009; 28: 319-33.

[4] Bauer G. Changes in physiological and biomechanical parameters by the Using a differential technique training in cycling. (germ. Änderungen von physiologischen und biomechanischen Parameter durch den Einsatz von differenziellem Training beim Radfahren). M.A. Thesis. Austria: Department of Sport and Movement Science, University of Salzburg 2007.

[5] Beckmann H, Schöllhorn WI. Differential training in shot put (germ. Differenzielles Lernen im Kugelstoßen). Leistungssport 2006; 36: 44-50.

[6] Wagner H, Müller E. The effects of differential and variable training on the quality parameters of a handball throw. Sports Biomech 2008; 7: 54-71.

[7] Schöllhorn WI, Michelbrink M, Welminski D, Davids D. Perspectives on cognition and action in sport. In: Araujo D, Ripoll H, Raab M, Eds. Hauppauge, NY: Nova Science 2009; pp. 59-73.

[8] Schöllhorn WI, Bauer HU. International Symposium on Biomechanics in Sports. In: Riehle H, Vieten M, Eds. XVI. Konstanz: Universitätsverlag 1998; pp. 574-7.

[9] Schöllhorn WI, Nigg BM, Stefanyshyn D, Liu W. Identification of individual walking patterns using time discrete and time continuous data sets. Gait Posture 2002; 15: 180-6.

[10] Bernstein NA. The coordination and regulation of movements. Oxford: Pergamon 1967.

[11] Hatze H. Motion variability - its definition, quantification and origin. J Mot Behav 1986; 18: 5-16.

[12] Schöner G, Haken H, Kelso JA. A stochastic theory of phase transitions in human hand movement. Biol Cybern 1986; 53: 24757.

[13] Mitra S, Riley MA, Turvey MT. Chaos in human rhythmic movement. J Mot Behav 1997; 29: 195-8.

[14] Körding KP, Wolpert DM. Bayesian decision theory in sensorimotor control. Trends Cogn Sci 2006; 10: 319-26.

[15] Körding KP, Tenenbaum JB, Shadmehr R. The dynamics of memory as a consequence of optimal adaptation to a changing body. Nat Neurosci 2007; 10: 779-86.

[16] Schöllhorn WI. Individuality - a neglected parameter? (germ. Individualität - ein vernachlässigter Parameter? ) Leistungssport 1999; 29: 7-11.

[17] Davids K, Chow, JY, Shuttleworth R. A constraints-based framework for nonlinear pedagogy in physical education. J Phys Educ NZ 2005; 38: 17-29.

[18] Chow JY, Davids K, Button C, Shuttleworth R, Renshaw I, Araujo D. The role of nonlinear pedagogy in physical education. Rev Educ Res 2007; 77: 251-78.

[19] Schöllhorn WI, Michelbrink M, Beckmann H, Trockel M, Sechelmann M, Davids K. Does noise provide a basis for the unification of motor learning theories? Int J Sport Psychol 2006; 37: 34-42.

[20] Shea JB, Morgan RL. Contextual interference effects on the acquisition, retention and transfer of a motor skill. J Exp Psychol Hum Learn Mem Cogn 1979; 5: 179-87.

[21] Schöllhorn WI, Sechelmann M, Trockel M, Westers R. Never train the right in order to become the best (germ. Trainiere nie das Richtige, um richtig gut zu werden). Leistungssport 2004; 4: 13-7.

[22] Trockel M. Differential training in football. (Germ. Differentielles Training im Fußball). B.Ed. Thesis. Germany: Department of Sport Science, University of Münster 2002.

[23] Bortz J, Lienert GA. Concise statistics for clinical research. $3^{\text {rd }}$ ed. Berlin: Springer 2008.

[24] Gentile AM. A working model of skill acquisition with application to teaching. Quest 1972; 17: 3-23. 
[25] Gigerenzer G, Hoff Rage U. How to improve Bayesian reasoning without instruction: frequency formats. Psychol Rev 1995; 102: 684-704.
[26] Hoffrage U, Gigerenzer G. Using natural frequencies to improve diagnostic inferences. Acad Med 1998; 73: 538-40.

(C) Schöllhorn et al.; Licensee Bentham Open.

This is an open access article licensed under the terms of the Creative Commons Attribution Non-Commercial License (http://creativecommons.org/licenses/ by-nc/3.0/) which permits unrestricted, non-commercial use, distribution and reproduction in any medium, provided the work is properly cited. 